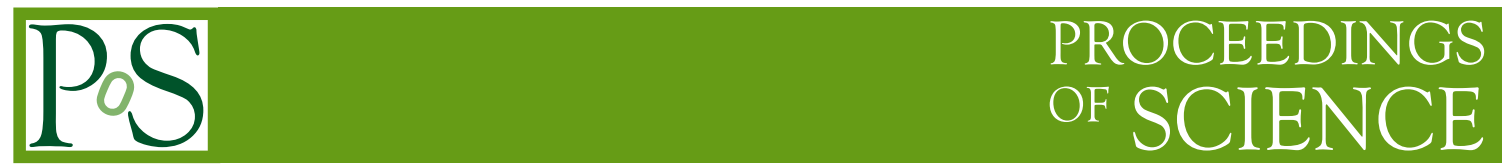

\title{
Searches for heavy particles with leptons at ATLAS
}

\author{
Federico Scutti ${ }^{1, *}$ \\ The University of Melbourne, \\ Parkville VIC 3010, Melbourne, Australia \\ E-mail: federico.scutti@unimelb.edu.au
}

Many theories beyond the Standard Model predict new phenomena, such as $Z^{\prime}, W^{\prime}$ bosons or heavy neutrinos, in final states with isolated high- $p_{\mathrm{T}}$ leptons $e / \mu / v$. Searches for new physics with such signatures, produced either resonantly or non-resonantly, are performed using the ATLAS experiment at the LHC. The recent $13 \mathrm{TeV}$ pp results will be reported.

40th International Conference on High Energy physics - ICHEP2020

July 28 - August 6, 2020

Prague, Czech Republic (virtual meeting)

${ }^{1}$ On behalf of the ATLAS collaboration.

${ }^{*}$ Speaker 
Numerous theories beyond the Standard Model of particle physics require the existence of high-mass particles featuring light-lepton $(e / \mu / v)$ decays observable from LHC [1] proton-proton collisions. The ATLAS experiment [2] has searched for these particles using event topologies requiring two isolated leptons, an isolated lepton and missing transverse energy, or requiring two leptons and jets in the final state. These searches feature datasets collected during Run 2 with integrated luminosities ranging from $\approx 36.1 \mathrm{fb}^{-1}$ to $139 \mathrm{fb}^{-1}$.

Two isolated same-flavour and oppositely-charged leptons are required for a resonant search [3], and a non-resonant one [4]. A resonant final state could stem from the decay of a heavy neutral spin-1 boson, like a $Z^{\prime}$ predicted in the Sequential Standard Model [5], E $_{6}$-motivated Grand Unification [6] or Heavy Vector Triplet [7] models. Also, spin-2 resonances are predicted in the Randall-Sundrum model [8] and spin-0 ones in the Minimal Supersymmetric Standard Model [9]. A resonant function is fitted to the di-lepton mass distribution, where a resonant shape is chosen for the signal and a smooth functional form for the background. Signal shapes are constructed from non-relativistic Breit-Wigner functions of various widths convolved with the detector resolution. The background functional form is based on fit performance studies on a simulated sample. Di-electron and di-muon selections are considered both as independent channels, and in a combined approach in the final exclusion limit setting under a lepton-flavour universality assumption [5, 6]. Final exclusion plots are shown in Figure 1.

A non-resonant signal is motivated by the hypothetical compositeness of quarks and leptons. This could manifest itself through an effective four-fermion contact interaction at energies well below the compositeness scale $\Lambda[10,11]$. The differential cross-section of the $q \bar{q} \rightarrow l^{+} l^{-}$contact interaction is parameterised by $\Lambda$, a purely contact interaction term, and an interference term with the Standard Model Drell-Yan process. The latter depends on the chiral left-handed or right-handed structure of the fermionic interaction. The model-independent search is set for a signal manifesting itself as a deviation from the expected gradient of the high-mass tail of the di-lepton mass spectrum. The background at high masses is extrapolated from a low-mass control region where the signal contribution is negligible and integrated in the single-bin signal region. The functional form of the background is determined using a data-driven approach. Signal distributions are obtained using a matrix-element weighting [12] of leading-order Drell-Yan samples. Various signal and control regions are optimised depending on the constructive or destructive nature of the signal interference term. Final exclusion limits are shown in Figure 2 in the more general model-independent approach, as well as also taking into account the chiral structure of the cross-section parameterisation. A further search has been performed by ATLAS for charged heavy spin-1 boson resonances decaying into an electron or muon plus a neutrino [13]. The existence of this particle, generally addressed as $W^{\prime}$, is hypothesised by several models [14-16] where the interpretation used in the search is the one provided by the Sequential Standard Model, where the $W^{\prime}$ couples to fermions with the same strength as the $W$ boson in the Standard Model but with suppressed coupling to the other bosons. Events are selected by requiring one isolated lepton and missing transverse energy in the event. The lepton momentum and the missing energy are used to compute the transverse mass of the event which is used as the main observable in the search. The background originated from jets mis-identified as leptons is modelled using a data-driven technique, while other backgrounds, mostly dominated by the Standard Model $W$ production, are modelled using simulation. For the final exclusion limits, the signal and background model of the transverse mass distribution is fitted to the 

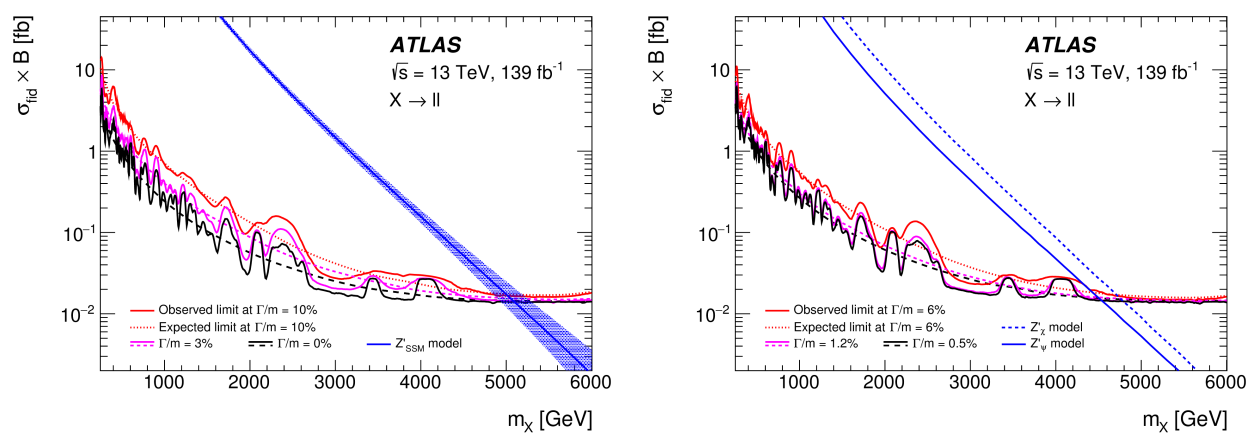

Figure 1: Upper limits at 95\% CL on the fiducial cross-section times branching ratio as a function of pole mass for the isolated di-leptons search [3]. The plots display limits obtained for various assumptions of the resonance width. Also shown are the theoretical cross-sections for the Sequential Standard Model $Z^{\prime}$ (left), and for a model motivated by Grand Unification (right). Theoretical uncertainties are shown as a band in the left plot. They are not included in the limit derivation.
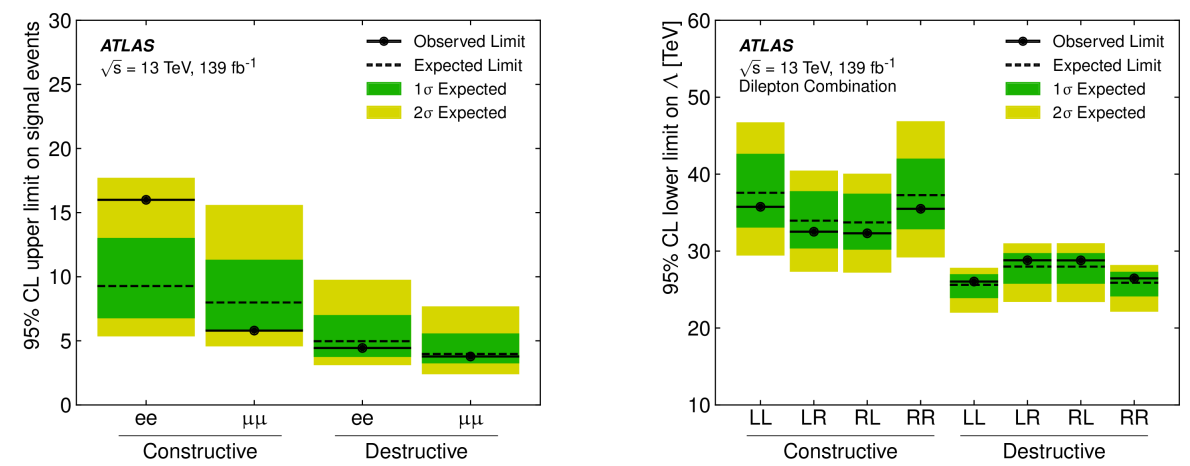

Figure 2: Left: model-independent upper limits at $95 \% \mathrm{CL}$ for the non-resonant di-lepton search [4] on the number of signal events for the constructive and destructive interference terms. Right: lower limits at $95 \%$ $\mathrm{CL}$ on $\Lambda$ obtained for the combination of the electron and muon channels and taking into account the chiral structure of the interaction in the interference term.

spectrum in data. Fiducial cross-section limits are provided for various width-over-mass ratios using a requirement removing the low-mass tail where background is enriched, as indicated in Figure 3 on the left, with respect to the limit obtained with the full transverse mass distribution, shown on the right. A search for pair production of the heavy leptons predicted by Type-III [17] See-Saw models [18] has also been performed [19]. The analysis looks for the decays of neutral states $N^{0}$ and charged ones $L^{ \pm}$with intermediate $W$ bosons and yielding two final state light-leptons of different flavour and charge combinations with at least two jets and large missing transverse momentum in the final state. Neutral and charged heavy lepton states are considered mass-degenerate and their mass $m\left(N^{0}, L^{ \pm}\right)$is the only free parameter of the model. The unknown mixing angles between the Standard Model leptons and the new heavy lepton states enter only in the expression of the $N^{0}$ and $L^{ \pm}$widths. The production cross-section does not depend on the mixing angles as heavy leptons are produced through couplings to electroweak bosons. Only the branching fractions of heavy leptons decays to a certain lepton flavour depend on the mixing angles. These branching ratios are considered to be equal for all three leptonic flavours and fixed to a value of $1 / 3$. The 

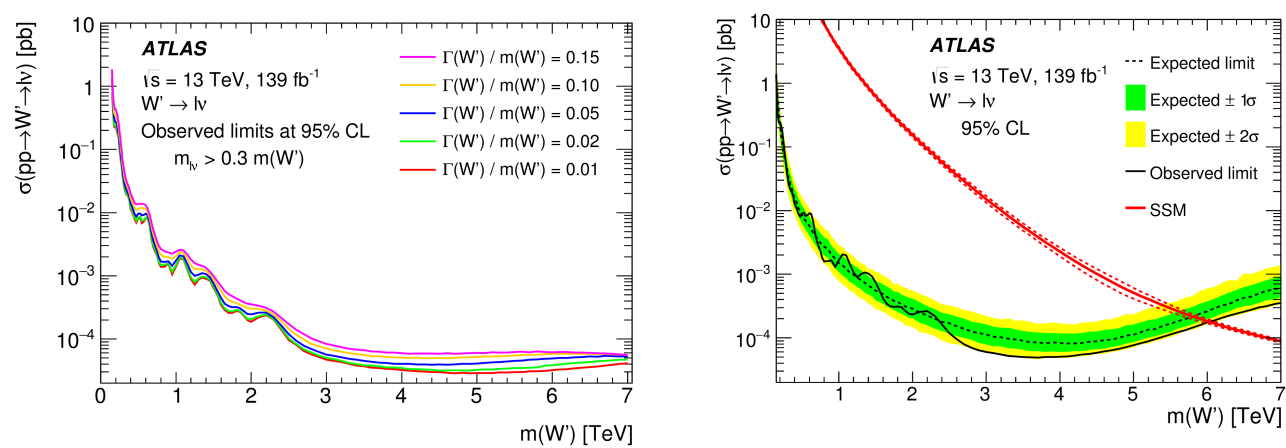

Figure 3: Left: limits on the fiducial cross-section for a number of different choices of width-over-mass ratios. Right: upper limits at 95\% CL on the $W^{\prime}$ production cross-section set in [13], in the framework of the Sequential Standard Model. Dashed lines surrounding the theoretical cross-section correspond to theoretical uncertainties.

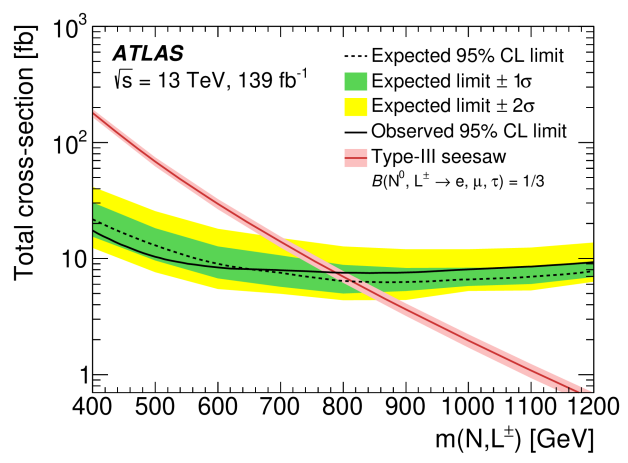

Figure 4: Exclusion limits at 95\% CL for the Type-III See-Saw heavy lepton search [19]. The model cross-section is shown in red.

search is optimised in six channels distinguished by flavour combination and charge-product of the final state lepton pair, where same-charge or opposite-charge pairs are considered. A large missing transverse energy significance is required of $\operatorname{Sig}\left(E_{\mathrm{T}}^{\mathrm{miss}}\right)>10$ for opposite-charge final states and $\operatorname{Sig}\left(E_{\mathrm{T}}^{\mathrm{miss}}\right)>7.5$ for same-charge ones. A boosted decay topology is exploited for decay products stemming from heavy leptons, requiring the combined transverse momentum for di-jet and lepton pairs to be above $100 \mathrm{GeV}$. The final exclusion limit is shown in Figure 4. Heavy right-handed Majorana or Dirac neutrinos $N_{\mathrm{R}}$ are searched for together with the heavy right-handed gauge bosons, $W_{R}$, predicted in the Left-Right Symmetric Model [20]. The search [21] is performed 

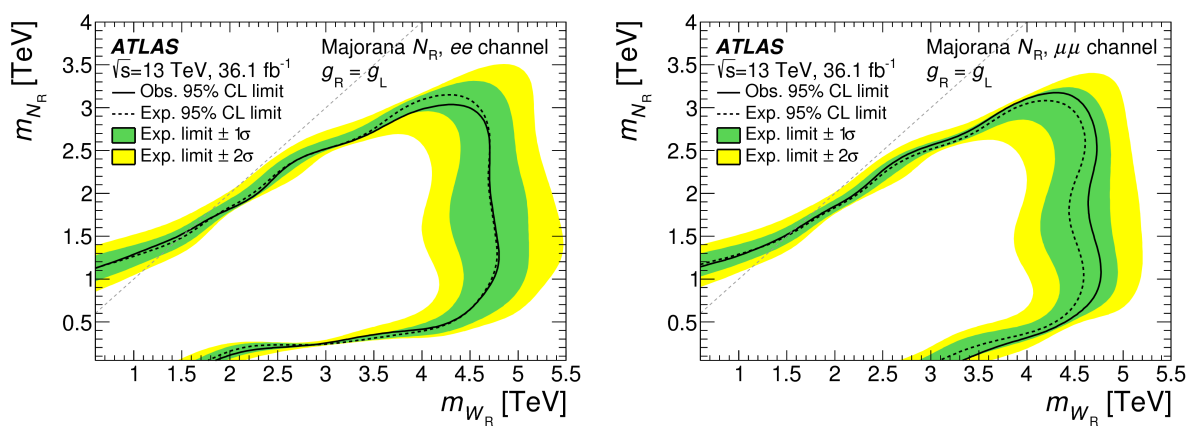

Figure 5: Exclusion limits from the search for heavy Majorana or Dirac neutrinos in the Left-Right Symmetric Model [21]. The area within the curves is excluded at 95\% CL. These limits refer to the hypothesis where the heavy neutrino is a Majorana particle. The dashed gray line indicates the region of the plane where $N_{\mathrm{R}}=W_{\mathrm{R}}$.

using a pair of two energetic electrons or muons in the final state which have either the same electric charge or opposite. Different charge combinations are used in independent selections which are also optimised separately for electrons and muons. The masses of the heavy neutrino and of $W_{R}$ are the free parameters taken into account. No flavour mixing is considered. In addition to this, left-handed and right-handed weak gauge couplings are defined to be equivalent. The final state contains two charged leptons and two jets. The production mechanism is the so-called KeungSenjanović process [22]. When the $W_{\mathrm{R}}$ mass is heavier than the $N_{\mathrm{R}}$ mass, the on-shell $W_{\mathrm{R}}$ mass is reconstructed from the invariant mass of the di-lepton and di-jet system, while, when the neutrino is heavier, only the di-jet mass is considered. The scalar sum between the transverse momenta of the selected leptons and the jets has to be larger than $400 \mathrm{GeV}$. The Majorana or Dirac nature of $N_{\mathrm{R}}$ is established by comparing the charges of the two leptons in the final state. If $N_{\mathrm{R}}$ is a pseudo-Dirac particle the leptons will always have opposite charge. However, if they are Majorana particles, the final state will contain opposite-charge and same-charge pairs with equal probability. Final exclusion plots are shown in Figure 5 under the assumption of Majorana $N_{\mathrm{R}}$. Corresponding plots for the pseudo-Dirac hypothesis yield similar results due to the similar sensitivities achieved by the opposite-charge and same-charge selection optimisations.

A different phase space is probed by the search for $W_{\mathrm{R}}$ with boosted $N_{\mathrm{R}}$ decays [23]. Electron or muon pairs are considered but no charge-product requirement is applied to them. Jets are reconstructed using a large radius algorithm, with radius parameter $R=1.0$, where the two jets in the final state are merged into one which is required to overlap with one light lepton, have a transverse momentum higher than $200 \mathrm{GeV}$, and be back-to-back in the transverse plane with respect to one recoiling lepton. Exclusion limits are shown in Figure 6.

\section{References}

[1] L. Evans and P. Bryant, JINST 3 (2008) S08001.

[2] ATLAS Collaboration, JINST 3 (2008) S08003.

[3] ATLAS Collaboration, Phys. Lett. B 796 (2019) 68. 

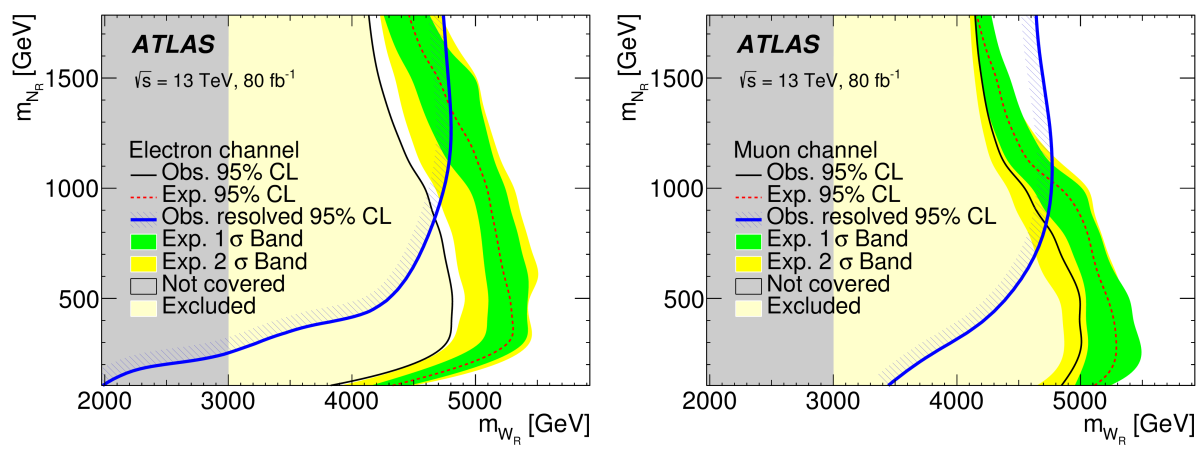

Figure 6: Exclusion limits at $95 \% \mathrm{CR}$ in the boosted decay topology search for $N_{\mathrm{R}}$ and $W_{\mathrm{R}}$ in the Left-Right Symmetric Model [23]. The blue line illustrates the comparison with the resolved topology search where two jets are required in the final state.

[4] ATLAS Collaboration, arXiv:2006.12946.

[5] G. Altarelli, B. Mele and M. Ruiz-Altaba, Z. Phys. C 47 (1990) 676.

[6] D. London and J. L. Rosner, Phys. Rev. D 34 (1986) 1530.

[7] F. del Aguila, J. de Blas and M. Perez-Victoria, JHEP 09 (2010) 033.

[8] L. Randall and R. Sundrum, Phys. Rev. Lett. 83 (1999) 3370.

[9] A. Djouadi, Phys. Rept. 459 (2008) 1.

[10] E. Eichten, I. Hinchliffe, K. D. Lane and C. Quigg, Rev. Mod. Phys. 58 (1986) 1065.

[11] E. Eichten, K. D. Lane and M. E. Peskin Phys. Rev. Lett. 50 (1983) 811.

[12] ATLAS Collaboration JHEP 2017 (2017) 182.

[13] ATLAS Collaboration, Phys. Rev. D 100, 052013 (2019).

[14] N. Arkani-Hamed, A. G. Cohen, E. Katz, and A. E. Nelson JHEP 07 (2002) 034.

[15] T. Appelquist, H.-C. Cheng, and B. A. Dobrescu Phys. Rev. D 64 (2001) 035002.

[16] K. Agashe, S. Gopalakrishna, T. Han, G.-Y. Huang, and A. Soni Phys. Rev. D 80, 075007.

[17] R. Foot, H. Lew, X. G. He, and G. C. Joshi, Z. Phys. C44 (1989) 441.

[18] R. N. Mohapatra and G. Senjanovic, Phys. Rev. Lett. 44 (1980) 912.

[19] ATLAS Collaboration, arXiv:2008.07949.

[20] G. Senjanović and R. N. Mohapatra, Phys. Rev. D 12 (1975) 1502.

[21] ATLAS Collaboration, JHEP 2019, 16 (2019).

[22] W.-Y. Keung and G. Senjanović, Phys. Rev. Lett. 50 (1983) 1427.

[23] ATLAS Collaboration, Phys. Lett. B 798 (2019) 134942. 